\title{
Homenaje de la Academia de Ciencias de Nicaragua a la Universidad Centroamericana en su 50 Aniversario*
}

\section{Carlos Tünnermann}

Academia de Ciencias de Nicaragua

Por acuerdo unánime de sus miembros, la Academia de Ciencias de Nicaragua, en su última Asamblea General, decidió rendir un homenaje a la Universidad Centroamericana, en ocasión de conmemorarse este año el Cincuenta Aniversario de su fundación.

Es justo y acertado que la Academia de Ciencias de nuestro país ofrezca este merecido tributo a una de las instituciones que más ha contribuido al cultivo, transmisión y difusión de las ciencias en Nicaragua.

La Universidad Centroamericana, sin lugar a dudas, desde sus primeros años de existencia, ha demostrado un constante compromiso con la investigación científica y su aplicación a la búsqueda de soluciones para nuestros problemas. Además, a este compromiso con la ciencia le ha impreso, desde un comienzo, una clara dimensión social.

En el quehacer de esta ilustre Casa de Estudios Superiores siempre han estado presentes las tres funciones básicas de la universidad moderna: docencia, investigación y extensión. Además, ha procurado que estas tres funciones contribuyan a la formación integral de sus graduados.

Como todos sabemos, sobre la simbiosis entre docencia e investigación -desde su formulación por Guillermo de Humboldt para la organización de la Universidad de Berlín (1810)- descansa la universidad moderna. Humboldt devolvió a la universidad tareas hasta entonces confiadas a las academias y sociedades científicas, señalándole como función la investigación y la formación de la persona. Sin embargo, la universidad de nuestros días no puede, sin caer en el aislamiento estéril, asumir, como recomendaba

Tomado del discurso del Dr. Carlos Tünnermann pronunciado en ocasión del homenaje de la Academia de Ciencias de Nicaragua a la Universidad Centroamericana en su 50 Aniversario. Ceremonia llevada a cabo en el Instituto de Historia de Nicaragua y Centroamérica (IHN-CA) el 17 de mayo de 2010. 
Humboldt, sus responsabilidades científicas prescindiendo de sus obligaciones sociales. La ciencia penetra ahora todos los procesos y no puede cultivarse buscando únicamente sus efectos formativos no utilitaristas, a como pedía el neohumanismo alemán. Mas, a esta corriente de pensamiento le corresponde el mérito de haber colocado la investigación científica en el corazón de las preocupaciones de la universidad, decisión que tanto contribuyó al desarrollo de la ciencia contemporánea.

La investigación científica y tecnológica -factor clave del proceso de transformación y desarrollo de la sociedad- no puede estar ausente de las tareas universitarias, menos aún en los países subdesarrollados, donde muchas veces sólo las universidades reúnen los recursos materiales y humanos indispensables para sustentar la actividad científica. No puede, entonces, la universidad limitarse a la simple transmisión del conocimiento, sino que debe también empeñarse en su adelanto y difusión. Esto lo entendió muy bien la Universidad Centroamericana desde sus primeros años de existencia.

Si bien la investigación en la universidad debe estar en simbiosis con la formación y el adiestramiento de las nuevas generaciones, no debe reducírsele a un papel subalterno en relación con la docencia, mucho menos cuando se trata únicamente de la docencia de las profesiones liberales. Esto sería perjudicial para el desenvolvimiento de la ciencia misma. En la universidad, la investigación tiene derecho a su lugar propio. Mas en interés de ella misma y de las tareas formativas que incumben a la educación superior, debe contribuir no sólo al descubrimiento de nuevas verdades sino también a la transmisión de los métodos que permiten tales hallazgos, lo que precisamente garantiza la continuidad de la búsqueda.

En la universidad es la investigación, nos dice Julián Marías, la que vivifica y fecunda todas las demás funciones. Por otra parte, la investigación universitaria no se concibe sin la divulgación de sus logros, de sus procedimientos y la crítica constante de sus resultados y sus aplicaciones. La imagen misma de la universidad contemporánea tiene mucho que ver con sus aportes al progreso de la ciencia y a la indagación de los problemas que se dan en su contexto social, para los que tienen la obligación ineludible de proponer soluciones. Esta afirmación es válida tanto para las universidades de los países avanzados como para las de los países menos avanzados.

Las naciones que se contentan con recibir la ciencia por correspondencia y se limitan a adaptar las tecnologías extranjeras, jamás podrán constituirse en sujetos de su propia historia. De ahí que en la médula de los esfuerzos encaminados a promover el desarrollo integral de un país se encuentran el esfuerzo destinado a la investigación científica y la formulación de una adecuada política de desarrollo científico, tecnológico y de innovación.

Una de las características de las sociedades contemporáneas es el papel central del conocimiento en los procesos productivos, al punto que el calificativo más frecuente que suele dárseles es el de sociedades del conocimiento. Asistimos a la emergencia de un nuevo paradigma económico-productivo en el que el factor más importante no es ya la disponibilidad de capital, mano de obra, materias primas o energía, sino el uso intensivo del conocimiento y la información. Las economías más avanzadas hoy día se basan en la mayor disponibilidad de conocimiento e información. Las ventajas comparativas dependen 
cada vez más del uso competitivo del conocimiento y de las innovaciones tecnológicas. Esta centralidad hace del conocimiento un pilar fundamental de la riqueza y el poder de las naciones.

El mejoramiento substancial de nuestra competitividad requiere conocimiento, tecnología, manejo de información, destrezas; significa elevar la calidad de nuestros sistemas educativos y la preparación de nuestros recursos humanos al más alto nivel posible y formar la "inteligencia científica" de nuestros países. La competitividad implica incorporar el progreso técnico a la actividad productiva, lo que supone una base científica. Hoy en día no sólo compiten los aparatos económicos y las empresas, sino también las condiciones sociales, los sistemas educativos y las políticas de desarrollo científico y tecnológico. En realidad, es la sociedad entera, el país mismo, quien compite y no sólo el sector productivo.

Otro aspecto importante al que cabe aludir, es el de la llamada responsabilidad social de las universidades. Hoy día, el tema de la responsabilidad social de las universidades ocupa un lugar prominente en el debate internacional. En el caso de nuestro país, pensamos que la universidad tiene el deber histórico e ineludible de asumir plenamente su responsabilidad social, para lo cual necesita integrarse a su sociedad nacional y promover un diálogo constructivo con todos sus sectores, priorizando los más desfavorecidos.

La universidad debe orientar su quehacer inspirada en el paradigma compendio del desarrollo humano, endógeno y sostenible, es decir, en un concepto de desarrollo concebido para nuestros propios países y realidades, basado en nuestras propias fuerzas productivas y potencialidades humanas y animado del propósito de ampliar las oportunidades de bienestar y de calidad de vida de nuestra gente, acorde con su dignidad humana y respetuoso de la naturaleza y el ambiente. Esto implica imprimir a su quehacer un sentido de pertinencia social, es decir, estar consciente de que la relevancia de su trabajo será evaluada en función de su auténtico compromiso social y de que éste genere beneficios concretos a su sociedad, priorizando el empoderamiento de los sectores excluidos o marginados.

La universidad deberá comprender que en países como los nuestros ella no puede sustraerse de la lucha contra la pobreza, la exclusión social, el desempleo, el analfabetismo y la superación de los grandes déficits que padecen nuestros pueblos en sus derechos humanos fundamentales. Todo esto debería conducirla a adoptar el modelo de "Investigación - Acción - Participación”, tal como lo propuso el sociólogo colombiano Orlando Fals Borda y es el que advierto en el quehacer científico de la UCA.

La reciente "Conferencia Mundial sobre Educación Superior”, auspiciada por la UNESCO (París, julio de 2009), confirmó la importancia y vigencia de estos conceptos, a nivel mundial, cuando proclamó que las universidades deben contribuir con sus investigaciones a enfrentar los grandes desafíos mundiales y asumir, resueltamente, su responsabilidad social. Reiteró, además, que la educación superior es "un bien público y un imperativo estratégico para todos los niveles de la educación y la base para la investigación, la innovación y la creatividad”. "Como nunca antes en la historia, dijo la Conferencia Mundial, es importante invertir en la educación superior como la mayor fuerza para construir sociedades del conocimiento inclusivas y diversas". 
Hemos creído conveniente recordar estos conceptos por cuanto en el desempeño de la UCA están presentes, principalmente en su trabajo de los últimos años, que le ha valido ocupar el primer lugar entre las 52 instituciones y centros de educación superior en Nicaragua y ubicarse entre las diez mejores a nivel centroamericano según el "Ranking Mundial de Universidades", que anualmente realiza el Consejo Superior de Investigaciones Científicas de España. Cabe destacar que este organismo toma en cuenta la visibilidad, publicaciones y calidad del contenido académico y científico, así como actividades de investigación y proyección social.

A este importante logro han contribuido todos los integrantes de la comunidad académica de la UCA, liderada en sus distintas etapas históricas por ilustres Rectores, desde su fundador, Padre León Pallais, S.J., pasando por el P. Arturo Divar, el Dr. Juan Bautista Arríen, el P. Amando López, el Padre Miguel Ángel Ruiz Vicario, Padre César Jerez, Padre Xabier Gorostiaga, Padre Eduardo Valdés y su actual Rectora, Dra. Mayra Luz Pérez Díaz.

En un país como el nuestro, donde la investigación científica no ha sido hasta hoy tarea socialmente prioritaria, y donde se invierte en actividades de ciencia y tecnología apenas el $0.07 \%$ del PIB, frente al 1\% que ya invierten otros países de la región -siguiendo una recomendación de la UNESCO formulada hace más de cincuenta años- sobresalen las tareas de cultivo de la ciencia y la investigación que realizan las universidades que forman parte del Consejo Nacional de Universidades, a tal punto que puede afirmarse que estas universidades constituyen la columna vertebral de las tareas de investigación en el país, ya que llevan a cabo cerca del $90 \%$ de esas tareas.

Y entre las universidades miembros del CNU, ocupa un lugar destacado la Universidad Centroamericana, tanto por el amplio panorama que abarcan sus labores de investigación como por la solidez de su contribución al adelanto de la ciencia en nuestro país. Efectivamente, el espectro de las investigaciones que la UCA lleva a cabo, abarca desde las Ciencias Sociales, las Ciencias Históricas y las Humanidades hasta las Ciencias Naturales y campos bien especializados, como lo es la Biología Molecular y la Malacología.

Sin que sea nuestro propósito ofrecer un exhaustivo inventario de ese valioso aporte, lo que demandaría un tiempo mucho mayor que el que se me ha asignado, voy a referirme en esta ocasión a los aspectos que considero más sobresalientes de ese aporte.

Los primeros institutos de investigación de la UCA fueron el Instituto de Historia de Nicaragua y Centroamérica, el Centro de Análisis Sociocultural y la Estación Solar Vadstena - UCA. A ellos se han agregado el Centro de Investigaciones de Ecosistema Acuáticos, el Centro de Malacología y Diversidad Animal, el Instituto de Investigación y Desarrollo Nitlapan, el Instituto de Educación de la UCA "Xabier Gorostiaga" y el Centro de Biología Molecular. Se trata de toda una constelación de Institutos y Centros dedicados a la investigación, el estudio de los problemas nacionales y la difusión científica.

Diremos unas pocas palabras sobre cada uno de ellos:

El Instituto de Historia de Nicaragua y Centroamérica, cuya creación debemos al Padre Álvaro Argüello Hurtado es, sin duda, uno de los institutos insignia de la UCA. Ha recibido varios 
reconocimientos, siendo el más reciente el "Premio Príncipe Claus", otorgado por la Fundación Príncipe Claus de los Países Bajos por su dedicación al rescate y enseñanza de la historia, su compromiso con la investigación y su aporte al fortalecimiento de la identidad cultural nicaragüense y la recuperación de la memoria de los pueblos indígenas. Es depositario de los archivos de la Cruzada Nacional de Alfabetización de 1980, incorporados al Programa Memoria del Mundo de la UNESCO. De todos es conocida la calidad de las publicaciones de este Instituto, la mayoría de ellas producto de sus investigaciones históricas y documentales, y de su "Revista de Historia”. De manera especial cabe señalar el aporte de este Instituto a la transformación y mejoramiento de la enseñanza de la historia de Nicaragua. En el campo de la proyección social, cómo no mencionar el "Tren Cultural: Memoria de la Cruzada Nacional de Alfabetización de 1980 en Nicaragua”, novedoso proyecto educativo y de proyección del patrimonio cultural, que ha tenido un gran impacto entre los jóvenes estudiantes del país. El Instituto es parte de una amplia red de institutos y centros de investigación y proyección social.

NITLAPAN es otro de los institutos más reconocidos de la UCA, especializado en la investigación, creación y difusión de nuevos modelos y metodologías de desarrollo local y urbano. En su creación fue decisivo el entusiasmo y compromiso de su fundador, el Padre Peter Marchetti, S.J. La investigación aplicada a la promoción del desarrollo socioeconómico y medioambiental constituye la columna vertebral de la estrategia institucional de Nitlapan, que también lleva a cabo proyectos de investigación conjunta con prestigiosas universidades y centros de investigación nacionales y extranjeros, sobre sistematización y evaluación de impacto de las iniciativas de desarrollo local; análisis y estudios sectoriales, nacionales y macroeconómicos cuyos informes trascienden el ámbito de la experiencia local.

El Centro de Análisis Sociocultural (CASC-UCA) fundado en 1983, es el resultado de la relación académica solidaria entre la UCA y la Universidad Católica de Lovaina (Bélgica) y fruto del énfasis en el compromiso social universitario que marcó la gestión del inolvidable Rector, Padre César Jerez, más el apoyo constante del sociólogo de la religión François Houtart. Fruto de sus 27 años de labor investigativa, formativa y de divulgación de temas relacionados con la transición cultural, cambios religiosos y desarrollo local en el país y la región centroamericana, así como de promover una cultura política comprometida con el desarrollo y la democracia participativa, el CASC-UCA ha publicado más de una veintena de libros y monografías relacionados con sus temas de estudio. El CASC ha sido fundador y miembro importante de iniciativas de redes y plataformas regionales comprometidas con la investigación y promoción del desarrollo, la gobernanza y la democracia a nivel nacional y regional, tales como la Red Nicaragüense por la Democracia y el Desarrollo Local, el Instituto para el Desarrollo Local de Centroamérica y la Mesa de la Descentralización de Nicaragua. Es miembro activo del Consejo Latinoamericano de Ciencias Sociales (CLACSO).

La Estación Solar "Vadstena - UCA" es un proyecto de investigación que se inició en 1983 con el objetivo de crear una base de datos solares y meteorológicos que ayuden a conservar y mejorar el medio ambiente. Producto de sus investigaciones es la publicación intitulada "Radiación solar y atmosférica en Managua" del Padre Julio López de la Fuente.

El Centro de Malacología y Diversidad Animal se estableció en 1977 por los Padres Jesuitas Adolfo y Julio López de la Fuente. Al inicio de sus actividades se concretó al estudio de los 
moluscos marinos pero, a partir de 1988, amplió su campo de estudio a los continentales, acuáticos y terrestres. Ha realizado un estudio exhaustivo de los moluscos continentales de la vertiente del Pacífico de Nicaragua. Entre otras labores de investigación ha reunido una colección científica de referencia de moluscos del país debidamente catalogada. Esta labor se ha hecho en estrecha colaboración con distintos museos, especialmente el Museo de Historia Natural de los Ángeles, la Universidad Estatal de Iowa, el Instituto INBIO y el Museo Nacional de Costa Rica. Se ha establecido la Estación de Biodiversidad Santa Maura en la nebliselva tropical de Jinotega, abierta a investigadores de la Ecología Tropical. Las investigaciones de este Centro han dado como resultado ejemplos de modelos estadísticos y matemáticos de apoyo a la docencia e instrumentos de medición del estado de la biodiversidad de Nicaragua.

El Instituto de Educación de la UCA “Xabier Gorostiaga, S.J.” creado por iniciativa del Dr. Juan Bautista Arríen y un valioso equipo de especialistas en educación, aplica el modelo de investigación-acción mediante una serie de proyectos en el campo de la investigación socioeducativa que se proponen incidir en la política educativa del país. Varios de sus trabajos sobre la problemática educativa abarcan toda la región centroamericana. Como resultado de sus investigaciones ha publicado más de veinte obras que hoy representan un referente indispensable para los estudiosos de la educación en el país y Centroamérica.

El Centro de Investigación de Ecosistemas Acuáticos (CIDEA-UCA), fue fundado en 1995 con la cooperación del gobierno del Japón. Este Centro tiene como principal tema de investigación la calidad de las aguas del Golfo de Fonseca y de los esteros, principalmente en la zona de Puerto Morazán por su importancia para la producción camaronera del país. $\mathrm{Su}$ labor comprende realizar investigación, extensión, asistencia técnica, servicios de laboratorio y capacitación. Asimismo, aúna esfuerzos en procesos informativos y educativos de la población de la cuenca del Estero Real para ayudar a mejorar los métodos y técnicas de producción, aumentar la eficiencia con prácticas ambientalmente amigables y mejorar los ingresos, elevando así el nivel de vida de la personas. A través de la investigación y la docencia, el CIDEA brinda apoyo formal a la industria y a la educación superior, también proyecta capacitaciones y asistencia técnica a cooperativas y sectores de la producción acuícola a fin de contribuir con el uso sostenible de los recursos naturales del país. El Centro se propone llegar a ser un centro de referencia regional.

El Centro de Biología Molecular (CBM), creado por iniciativa del Dr. Jorge Huete, Presidente de nuestra Academia, se estableció en la Facultad de Ciencia, Tecnología y Ambiente de la Universidad Centroamericana (UCA) en 1999, con fondos y donaciones de la Fundación New England Biolabs, la OMS, la Universidad de California de San Francisco, la Fundación Pew y otros fondos privados y propios de la Universidad Centroamericana. Se convirtió en el primer centro de investigación molecular del país. El personal del Centro está conformado por investigadores con perfil de biología molecular, bioanálisis clínico, bioquímica, genética e ingeniería ambiental, que trabajan junto a estudiantes y pasantes. El objetivo central del CBM es impulsar la investigación y educación científica en Nicaragua y promover la aplicación de la ciencia en la solución de problemas locales de índole ambiental, agrícola y de salud, contribuyendo al desarrollo humano, económico e industrial. Desde 1999 el CBM ha venido creciendo en tamaño y áreas de trabajo. Actualmente 
provee servicios tecnológicos tales como diagnóstico forense por ADN, de utilidad para la policía y el sistema judicial, pruebas de paternidad para casos privados y agencias públicas como el Ministerio de la Familia, bioprospección de enzimas industriales y de interés a la salud, capacitaciones de nivel de posgrados y universitario, consultoría en biotecnología agrícola incluyendo alimentos y plantas transgénicas. El Centro divulga sus investigaciones a través de seminarios, talleres y publicaciones científicas. Además, organiza actividades científicas como los Congresos Nacionales de Biotecnología, contando con la participación de prestigiosos científicos nacionales y extranjeros y premios Nobel, con lo cual ha ganado un rol de liderazgo en la formación de políticas científicas y de desarrollo.

Finalmente, cómo no hacer aquí mención de las dos prestigiosas revistas de la UCA: “ENCUENTRO”, con 41 años de existencia y 85 números publicados y ENVÍO, con 29 años y 337 números de ininterrumpida circulación.

Como una "aventura del espíritu” calificó José Coronel Urtecho, en su célebre discurso inaugural de la UCA, la fundación de esta Universidad. Pero precisó: "Esta aventura del espíritu que es la Universidad no lo sería si no despertara en los estudiantes el espíritu de aventura”. ¿Y qué mejor y más maravillosa aventura que la investigación científica que nos conduce al descubrimiento de nuevas verdades? Ese es el reto que todos tenemos: despertar en nuestros jóvenes el espíritu de indagación, la curiosidad, el afán de aprender y la afición al estudio para que sigan aprendiendo durante toda su vida.

En nombre de la Academia de Ciencias de Nicaragua felicito, con mucho entusiasmo, a la Universidad Centroamericana por su medio siglo de fecunda existencia y formulo mis mejores votos porque su trayectoria, que espero sea de siglos, siga iluminando los destinos de Nicaragua.

Managua, 17 de mayo de 2010. 